in which $q_{1}$ is the velocity magnitude in the irrotational flow corresponding to the new complex potential $w^{\prime 2} / 2$. But it has been proved that the maximum value of $q_{1}^{2}$ in $D$ occurs on its boundary, hence the same is true for $a^{2}$.

Since $w^{\prime} w^{\prime \prime}$ is analytic in $D$, the result for the acceleration could have been reached through

$$
a^{2}=w^{\prime} w^{\prime \prime}\left(w^{\prime} w^{\prime \prime}\right)^{*}
$$

in (8) by means of the well-known maximum-modulus theorem for analytic functions, without resorting to $q_{1}$. However, since the corresponding result for the speed is often reached without the use of the maximum-modulus theorem, the present proof may seem preferable to some.

As a by-product of the proof, a means of evaluating the magnitude of the acceleration at any point in the field of flow is provided by (8), once $w(z)$ is known. Furthermore, since the acceleration is proportional to the gradient of the dynamic pressure, it follows from the present result that the maximum dynamic-pressure gradient in $D$ must occur on its boundary.

The boundary of $D$ is, of course, not necessarily a solid boundary. However, for ambiently uniform flows past arbitrarily shaped bodies, the maximum acceleration (or the maximum dynamic-pressure gradient) must occur on one of the solid boundaries. This can be seen by taking $D$ as the entire domain outside the solid bodies. Its boundary then consists of the solid boundaries and a boundary at infinity. Since the acceleration at infinity is zero, the maximum acceleration must occur on one of the solid boundaries.

\title{
AN ESTIMATE OF THE ERROR DUE TO THE TRUNCATED BOUNDARY IN THE NUMERICAL SOLUTION OF THE BLASIUS EQUATION
}

\author{
By L. A. RUBEL' (U. S. Naval Proving Ground*, Dahlgren, Va.)
}

We seek a numerical solution of the Blasius differential equation:

$$
f^{\prime \prime \prime}(x)+f(x) f^{\prime \prime}(x)=0
$$

satisfying the split conditions

$$
0=f(0)=f^{\prime}(0), \quad f^{\prime}(\infty)=2 .
$$

The condition $f^{\prime}(\infty)=2$ is clearly unsuited for numerical procedures. In previous tabulations (e.g. [1], [2], [3]), the condition $f^{\prime}(\infty)=2$ is replaced by $f^{\prime}(M)=2$ where $M$ is chosen so large that the second derivative becomes indistinguishable from zero, and further integration becomes impossible. That this procedure yields, in any sense, an approximation to the desired function has not, to the author's knowledge, been established heretofore.

This paper demonstrates that for large $M$, the numerical solution lies close to the true solution, gives a computable estimate of the deviation from the true solution, and provides an a priori criterion by which any desired accuracy may be achieved.

${ }^{1}$ Received Nov. 12, 1954. The author is now at Cornell University.

*The opinions and assertions expressed herein are the private ones of the writer and are not to be construed as official or reflecting the views of the Navy Department or the Naval Establishment at large. 
Let $f(x)$ satisfy

$$
f^{\prime \prime \prime}+f f^{\prime \prime}=0, \quad f(0)=f^{\prime}(0)=0, \quad f^{\prime}(\infty)=2 .
$$

Let $g_{M}(x)=g(x)$ satisfy

$$
g^{\prime \prime \prime}+g g^{\prime \prime}=0, \quad g(0)=g^{\prime}(0)=0, \quad g^{\prime}(M)=2 .
$$

We observe some well known properties of $f(x)$; namely that $f^{\prime \prime}(x)>0$ for $x>0$; and therefore, that $f^{\prime}(x)$ increases steadily from 0 at $x=0$ to 2 at $x=\infty$. This may be demonstrated by observing that since $f^{\prime}(0)=0$ and $f^{\prime}(t)>0$ for some $t>0, f^{\prime}(x)$ must somewhere be an increasing function of $x$, and that $f^{\prime \prime}(x)$ must somewhere be positive.

If $f^{\prime \prime}(x)$ is negative for some positive $x$, then $f^{\prime \prime}\left(x_{0}\right)$ must be zero for some positive $x_{0}$. From the differential relation

$$
f^{\prime \prime \prime}\left(x_{0}\right)+f\left(x_{0}\right) f^{\prime \prime}\left(x_{0}\right)=0
$$

we see that $f^{\prime \prime \prime}\left(x_{0}\right)=0$. Repeated differentiation of the differential equation demonstrates that $f^{(n)}\left(x_{0}\right)=0$ for $n \geq 2$, and that, since $f$ is analytic at $x_{0}, f(x)$ is a polynomial, which is impossible.

The function $k^{2} f^{\prime}(k M)$ is zero for $k=0$, increases with $k$, and is unbounded as $k$ approaches $\infty$. For some value of $k$, then,

$$
k^{2} f^{\prime}(k M)=2 .
$$

For this value of $k$, we see that $g(x)=k f(k x)$ since $k f(k x)$ satisfies the requirements that determine $g(x)$ uniquely. It is clear that $k>1$.

Put $g^{\prime}(\infty)=2+\epsilon(M)=2 k^{2}$ so that $k^{2}-1=\frac{1}{2} \epsilon(M)$.

Let us now estimate $|g(x)-f(x)|$ for $x \leq M$.

$$
|g(x)-f(x)|=|k f(k x)-f(x)| \leq|(k-1) f(k x)|+|f(k x)-f(x)| .
$$

Since $f(0)=0$ and $f^{\prime}(x) \leq 2$ for $x \geq 0$, we have

$$
f(k x) \leq 2 k x \text {. }
$$

By the law of the mean of differential calculus,

$$
f(k x)-f(x)=f^{\prime}(\xi)(k-1) x \quad \text { for some } \xi, \quad x \leq \xi \leq k x .
$$

Since $f^{\prime}(\xi) \leq 2$,

$$
|f(k x)-f(x)| \leq 2(k-1) x .
$$

Hence,

$$
|g(x)-f(x)| \leq 2 k(k-1) x+2(k-1) x=2\left(k^{2}-1\right) x .
$$

But $k^{2}-1=\frac{1}{2} \epsilon(M)$, so that

$$
|g(x)-f(x)| \leq x \epsilon(M) \leq M \epsilon(M) \quad \text { for } \quad x \leq M .
$$

We now estimate $\epsilon(M)$.

$$
\epsilon(M)=g^{\prime}(\infty)-g^{\prime}(M)=\int_{M}^{\infty} g^{\prime \prime}(x) d x .
$$


Since

or

$$
\frac{g^{\prime \prime \prime}(x)}{g^{\prime \prime}(x)}=-g(x), \quad \log \frac{g^{\prime \prime}(x)}{g^{\prime \prime}(M)}=-\int_{M}^{x} g(x) d x
$$

$$
g^{\prime \prime}(x)=g^{\prime \prime}(M) \exp \left[-\int_{M}^{x} g(x) d x\right] .
$$

Now $g(x) \geq g(M)$ for $x \geq M$, so that

$$
\begin{gathered}
\int_{M}^{x} g(x) d x \geq g(M)(x-M), \\
\int_{M}^{\infty} g^{\prime \prime}(x) d x=g^{\prime \prime}(M) \int_{M}^{\infty} \exp \left[-\int_{M}^{x} g(t) d t\right] d x \\
\leq g^{\prime \prime}(M) \int_{M}^{\infty} \exp [-g(M)(x-M)] d x=\frac{g^{\prime \prime}(M)}{g(M)} .
\end{gathered}
$$

Hence

so that

$$
\epsilon(M) \leq \frac{g^{\prime \prime}(M)}{g(M)},
$$

$$
|f(x)-g(x)| \leq M \frac{g^{\prime \prime}(M)}{g(M)} \quad \text { for all } x \leq M .
$$

Notice that this estimate involves only the tabulated function $g$ and its derivatives so that the degree of approximation is directly computable. For example, the Emmons and Leigh tabulation [1] for $M=5$ yields

so that

$$
g_{s}^{\prime \prime}(5)=0 \pm 10^{-5}, \quad g_{5}(5)=8.27922 \pm 10^{-5}
$$

$$
\left|f(x)-g_{5}(x)\right| \leq \frac{5}{8} 10^{-5}, \quad(x \leq 5) .
$$

It is possible to estimate beforehand how large $M$ should be chosen to attain any desired accuracy, using the Emmons and Leigh tabulation.

Let $h(x)$ satisfy

$$
\begin{gathered}
h^{\prime \prime \prime}(x)+h(x) h^{\prime \prime}(x)=0, \\
h(0)=h^{\prime}(0)=0, \quad h^{\prime}(5)=2 .
\end{gathered}
$$

Put $h(x)=m f(m x)$.

Let $g(x)$ satisfy

$$
\begin{aligned}
g^{\prime \prime \prime}(x)+g(x) g^{\prime \prime}(x) & =0, \\
g(0)=g^{\prime}(0)=0, \quad g^{\prime}(M) & =2 \quad(M>5) .
\end{aligned}
$$

Put $g(x)=k f(k x)$. Then

$$
\begin{gathered}
g(x)=\frac{k}{m} h\left(\frac{k}{m} x\right) \\
|g(x)-f(x)| \leq M \frac{g^{\prime \prime}(M)}{g(M)}=M \frac{k^{2}}{m^{2}} \frac{h^{\prime \prime}(k M / m)}{h(k M / m)} .
\end{gathered}
$$


The tabulated values state:

$$
\begin{aligned}
h^{\prime}(1) & =1.25953 \pm 10^{-5}, \\
h^{\prime \prime}(0) & =1.32823 \pm 10^{-5}, \\
h^{\prime \prime}(5) & =0 \pm 10^{-5}, \\
h(5) & =8.27922 \pm 10^{-5}, \\
\frac{h^{\prime \prime}(5)}{h(5)} & \leq \frac{1}{8} 10^{-5} .
\end{aligned}
$$

Now $m^{2}-1=\frac{1}{2} \epsilon(M) \leq \frac{1}{16} 10^{-5}$ and $m \leq 1+10^{-6}$. Also $1<k<m$.

To estimate $h(k M / m)$ and $h^{\prime \prime}(k M / m)$, use the crude estimation:

$$
h(x) \geq h^{\prime}(1)(x-1)
$$

and the representation

$$
h^{\prime \prime}(x)=h^{\prime \prime}(0) \exp \left[-\int_{0}^{x} h(t) d t\right] \leq h^{\prime \prime}(0) \exp \left[-\int_{1}^{x} h(t) d t\right] .
$$

After certain manipulations, which may be far from the best, the author has arrived at the following result:

if $M \geq 1+2(N+1)^{1 / 2}$, then

$$
|g(x)-f(x)|<10^{-N} \quad \text { for } x \leq M .
$$

Hence, $M=8$ gives at least 10-place accuracy, and $M=10$ gives at least 19-place accuracy.

These last estimates are all crude. Once $M$ is chosen, however, and the tabulation is made, $M g^{\prime \prime}(M) / g(M)$ can be calculated easily, and measures the accuracy well.

\section{REFERENCES}

1. H. W. Emmons and D. Leigh, Tabulation of the Blasius function with blowing and suction, Harvard University, Division of Applied Sciences, Combustion Aerodynamics Laboratory, Interim Technical Report No. 9

2. John C. Martin, On Magnus effects caused by the boundary layer displacement thickness on bodies of revolution at small angles of attack, Ballistic Research Laboratory, Report No. 870

3. H. Schlichting, Boundary layer theory, Part I, Laminar Flows, NACA TM 1217, 1949

\section{ON THE EQUATIONS OF LINEARIZED CONICAL FLOW*}

By J. H. GIESE (Aberdeen Proving Ground, Maryland)

In an irrotational flow field with velocity potential function $\Phi\left(x, y, z^{\prime}\right)$ let $u, v, w^{\prime}$ be velocity components at the point with rectangular coordinates $x, y, z^{\prime}$. If $u, v, w^{\prime}$ are constant on rays through the origin the field is said to be conical [1,2]. Then $u, v, w^{\prime}$ are homogeneous of order zero in $x, y, z^{\prime}$, and $\Phi$ is homogeneous of order one if an unimportant arbitrary additive constant is neglected. Furthermore, if the flow field arises by slightly perturbing a uniform flow parallel to the $z^{\prime}$ axis at Mach number $M$, then

*Received August 30, 1954. 